\title{
Using of spectral analysis of heart rate variability for increasing reliability of bicycle ergometry results
}

\author{
Vladimir Ivanovich Gridnev, Anton Robertovich Kiselev, Olga Mikhailovna Posnenkova, \\ Vladimir Alexandrovich Shvartz
}

Centre of New Cardiological Informational Technologies, Saratov Research Institute of Cardiology, Saratov, Russia; antonkis@1ist.ru, schaen@,rambler.ru

Received 18 November 2010; revised 25 April 2011; accepted 18 June 2011.

\begin{abstract}
The aim was to study the suitability of the heart rate variability (HRV) spectral parameters for evaluations of bicycle ergometry results in coronary heart disease (CHD) patients. Methods. Our study included 243 male CHD patients aged $49 \pm 8$ years. The coronary atherosclerosis was assessed by coronary angiography. The results of bicycle ergometry, Doppler echocardiography and HRV spectral analysis were also analyzed. The duration of each stage of bicycle ergometry was $3 \mathrm{~min}$, the initial load value was $25 \mathrm{~W}$. Dynamic load continued until the patient had reached $75 \%$ of heart rate from his maximal age level. The maximal level of load achieved (i.e. load tolerance) was taken into consideration. We calculated sensitivity (Se), specificity $(\mathrm{Sp})$, likelihood ratios of positive (LR+) and negative (LR-) bicycle ergometry results. Results. All patients had similar clinical characteristics. LR+ become maximal under the moderate load tolerance. LR- are maximal in the CHD patients with high load tolerance. Thus, the excessiveness of false-negative results of bicycle ergometry is in CHD patients with high load tolerance. Reliability of results of bicycle ergometry increased under using assessments of low-frequency (LF) range power of HRV spectrum. Conclusions. Thus, the using of LF range power of HRV spectrum increases reliability of bicycle ergometry (or other load tests) results in CHD patients.
\end{abstract}

Keywords: Heart Rate Variability; Bicycle Ergometry; Coronary Heart Disease

\section{INTRODUCTION}

Submaximal exercise tests are most frequently used in diagnosis of coronary heart disease (CHD). But the sen- sitivity (Se) and specificity (Sp) of these kind of tests are limited [1]. Defining the conditions under which exercise tests may produce both true and false results, is an important task of applied cardiology.

It was shown that the heart rate variability (HRV) parameters turned out to be sensitive indicator of a myocardial and coronary status in CHD patients $[2,3]$. The model of heart control based on a baroreflex feedback loop [4-8] has great importance for researches of autonomic heart control. This model explains an origin of low-frequency (LF) component of HRV [9-15]. Thus, LF range of HRV spectrum is of the interest for evaluation of autonomic heart control in CHD patients during load tests.

The aim was to study the suitability of HRV spectral parameters for evaluations of bicycle ergometry results in CHD patients.

\section{METHODS}

\subsection{Subjects}

The study was approved by the Ethics Committee of the Saratov Research Institute of Cardiology in Saratov, Russia, and informed consent was obtained from all participants. Our study included 243 male CHD patients aged $49 \pm 8$ years. 132 CHD patients had acute myocardial infarction six months prior to the start of the study. Pathological Q-wave on electrocardiogram (ECG) was observed in $67 \mathrm{CHD}$ patients. Angina pectoris of I-II functional classes was observed in $86 \mathrm{CHD}$ patients and 120 CHD patients had angina pectoris of III-IV functional classes. $59 \mathrm{CHD}$ patients had arterial hypertension.

We excluded from the study CHD patients with renal pathology, valves heart disease, rhythm and conduction disorders hindering the HRV analysis.

\subsection{Measurements and Protocol}

The coronary atherosclerosis was assessed by selec- 
tive coronary angiography in all CHD patients. The severity of coronary atherosclerosis was assessed in according with the degree of maximal stenosis of coronary arteries (no less 75\%). Bicycle ergometry results, Doppler echocardiography results and HRV spectral analysis were also estimated.

Systolic and diastolic sizes of heart left ventricle, ejection fraction of the left ventricle (EF), the velocity of circumferential fiber shortening $\left(\mathrm{V}_{\mathrm{cf}}\right)$, isovolumetric relaxation time (IRT) and the condition of the left ventricle local contractility were determined by Doppler echocardiography.

Bicycle ergometry were performed by stepwise increasing load under electrocardiography (ECG) monitoring. The initial load was $25 \mathrm{~W}$. Bicycle ergometry continued till each CHD patient reached $75 \%$ of maximal heart rate level [16]. In case of ST segment depression no less $2 \mathrm{~mm}$ in one ECG lead during bicycle ergometry, this load test results were considered to be positive. Bicycle ergometry result was considered negative if CHD patient either reached $50 \%$ of maximal heart rate level at the highest point of load or performed load of no less $150 \mathrm{~W}$ during 3 minutes without any criteria of test cessation. CHD patients with other causes of test cessation were not included in this study. The maximal level of load achieved (i.e. load tolerance) was assessed. All CHD patients have stopped the treatment with nitrates ( 24 hours prior) and beta-blockers ( 3 - 7 days prior) before bicycle ergometry.

ECG recorded during $3 \mathrm{~min}$ both for rest and load of $25 \mathrm{~W}$ during bicycle ergometry. ECG signal was sampled at $250 \mathrm{~Hz}$ and digitized at 14 bits. For further analysis were only ECG and PPG records without artifacts, extrasystoles and considerable trends were left. ECG signal had been recorded between 13 and 15 hours.

\subsection{Statistical and Data Analysis}

Spectral characteristics of HRV were calculated using parametric method of spectrum estimation based on 14 order autoregression model construction. High-frequency (HF) range, $0.15-0.4 \mathrm{~Hz}$, and LF range, $0.04-0.15 \mathrm{~Hz}$, of HRV were analysed [17].

Sensitivity (Se), specificity (Sp), likelihood ratios of positive $(\mathrm{LR}+)$ and negative (LR-) bicycle ergometry results were estimated.

For a statistical analysis the software package Statistica 6.1 was used. We apply the Shapiro-Wilk test to check whether the HRV spectral data are approximately normally distributed. Since these data occur to be nonnormal their further analysis was carried out using nonparametric statistical methods. To compare the variables we used the Mann-Whitney test. Continuous variables are reported as medians $(\mathrm{Me})$ with inter-quartile ranges
$(25 \%, 75 \%)$. Categorical data are presented as frequencies and percentages. The obtained estimations were considered statistically significance if $\mathrm{P}<0.05$.

\section{RESULTS AND DISCUSSION}

CHD patients have been classified in two groups, according to bicycle ergometry results:

- First group included 125 CHD patients (aged $50 \pm 7$ years) with positive bicycle ergometry results,

- Second group included 118 CHD patients (aged $47 \pm$ 8 years) with negative bicycle ergometry results.

It was shown that both CHD patient's groups had similar clinical characteristics (age, sex, frequency of myocardial infarction, arterial hypertension, EF, systolic and diastolic sizes of heart left ventricle, etc.).

The group of CHD patients with positive bicycle ergometry results differed from the group with negative results in following characteristics: 1) less load tolerance by $36 \%(\mathrm{P}<0.001) ; 2)$ more clinically severe angina pectoris: $56 \%$ of CHD patients had III-IV functional class of angina and only $30 \%$ patients had angina of I-II functional class (versus $36 \%$ and $42 \%$ respectively in CHD patients with the negative bicycle ergometry results).

For the further analysis all CHD patients were divided into three groups according to the load tolerance level: low level $(<75 \mathrm{~W})$, moderate level $(75-125 \mathrm{~W})$, high level $(>125 \mathrm{~W})$. It is theoretically supposed that the load tolerance correlates with coronary atherosclerosis degree. These groups of patients had similar clinical characteristics. Frequency of positive bicycle ergometry results was greater in group of CHD patients with low load tolerance in comparison with other groups $(\mathrm{P}=$ 0.003 ). Angina was principal cause of bicycle ergometry cessation in the group of patients with low tolerance. Frequency of ECG ischemic changes was greater in the group of CHD patients with high tolerance $(77 \%)$ than in groups with moderate (64\%) and low (41\%) tolerance levels.

Frequency of significant coronary arteries stenosis decreased reliably under increase of the load tolerance in these patients groups (Table 1). In the group of CHD patients with high load tolerance, frequency of significant coronary arteries stenosis decreased 1.7 times $(\mathrm{p}<$ 0.001 ) in compare with the group with low tolerance, and it decreased in 1,5 times $(p=0.02)$ in group with high tolerance compared with group of patients with moderate tolerance $(\mathrm{p}=0.03)$. It is shown that $\mathrm{Se}$ of bicycle ergometry worsens under the decrease of load tolerance in CHD patients (Table 1).

Spectral analysis of HRV for the rest and load of $25 \mathrm{~W}$ displayed reliable differences between the groups of CHD patients with positive and negative bicycle er- 
Table 1. Coronary atherosclerosis in CHD patients with different load tolerance. Dependence of bicycle ergometry sensitivity (Se) and specificity ( $\mathrm{Sp}$ ) from load tolerance in these patients.

\begin{tabular}{lccc}
\hline Indices & $\begin{array}{c}\text { Low tolerance } \\
\text { level } \\
(<75 \mathrm{~W}) \\
\mathrm{n}=54\end{array}$ & $\begin{array}{c}\text { Moderate } \\
\text { tolerance level } \\
(75-125 \mathrm{~W}) \\
\mathrm{n}=113\end{array}$ & $\begin{array}{c}\text { High } \\
\text { tolerance level } \\
(>125 \mathrm{~W}) \\
\mathrm{n}=59\end{array}$ \\
\hline $\begin{array}{l}\text { Coronary artery } \\
\text { stenosis, } \\
\text { \% of CHD patients }\end{array}$ & 78 & 71 & 47 \\
Se, \% & 93 & 69 & 29 \\
$\mathrm{Sp}, \%$ & 25 & 79 & 81 \\
\hline
\end{tabular}

gometry results (Figure 1). CHD patients with negative test results had level of autonomic heart control differing from CHD patients with positive results in presence of similar clinical and instrumental status and coronary atherosclerosis. LF and HF ranges power of HRV spectrum was greater in CHD patients with the negative bicycle ergometry results than in other groups.

Load tolerance and HRV spectral parameters correlated with negative bicycle ergometry results under rest in CHD patients; multiple correlation coefficient was $0,41(p=0,004)$. In group of CHD patients with positive test results there was no correlation between the HRV spectral parameters and load tolerance $(\mathrm{P}=0.26)$.

These distinctions of LF range became a basis for improvements of diagnostic effectiveness of bicycle ergometry. Using the load of $25 \mathrm{~W}$ allows supposing autonomic heart control to be in some functional activity. This fact is a way of condition standardization for using of HRV spectral analysis. Three ranges of LF power were chosen in all CHD patients. They were the following for the rest: $<200 \mathrm{~ms}^{2}, 200-400 \mathrm{~ms}^{2},>400 \mathrm{~ms}^{2}$. And they were the following for $25 \mathrm{~W}$ load: $<75 \mathrm{~ms}^{2}, 75-$ $150 \mathrm{~ms}^{2},>150 \mathrm{~ms}^{2}$. The range intervals correspond to $33 \%$ and $66 \%$ percentiles of values in the sample of the examined patients, i.e. about one third of all CHD patients are presented in each group. It is shown that bicycle ergometry is of the most diagnostic value under $\mathrm{LF}_{\text {rest }}$ $>400 \mathrm{~ms}^{2}$ and $\mathrm{LF}_{25 \mathrm{w}}>150 \mathrm{~ms}^{2}$. In these cases, sum of $S e$ and $S p$ is maximal (Figure 2). Thus, for increase a reliability of bicycle ergometry in CHD patients It is possible to use LF range spectral power, it is applied especially for positive bicycle ergometry results (Figure 3). This concerns both standard bicycle ergometry procedure and analysis of bicycle ergometry results taking into account load tolerance (Tables 2 and 3). Positive results of bicycle ergometry in CHD patients are more reliable under moderate load tolerance and $L_{25} \mathrm{w}>$ $150 \mathrm{~ms}^{2}$. Otherwise a diagnostic effectiveness of bicycle ergometry decreases. Reliability of negative bicycle ergometry results is maximal under high load tolerance, independently of LF range power of HRV spectrum.

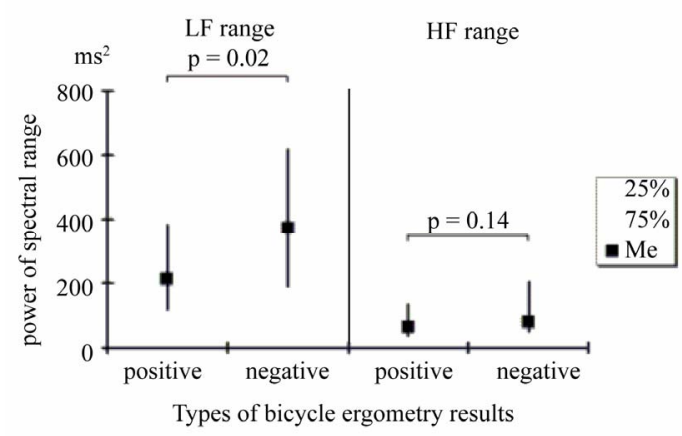

(a)

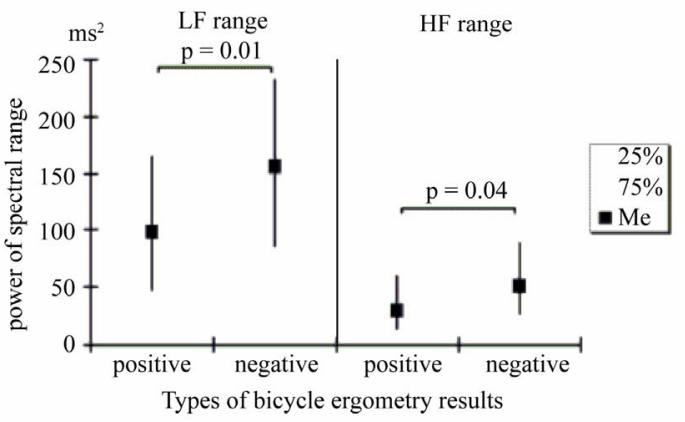

(b)

Figure 1. HRV indices in CHD patients with positive and negative bicycle ergometry results in rest (a) and load of $25 \mathrm{~W}$ (b).

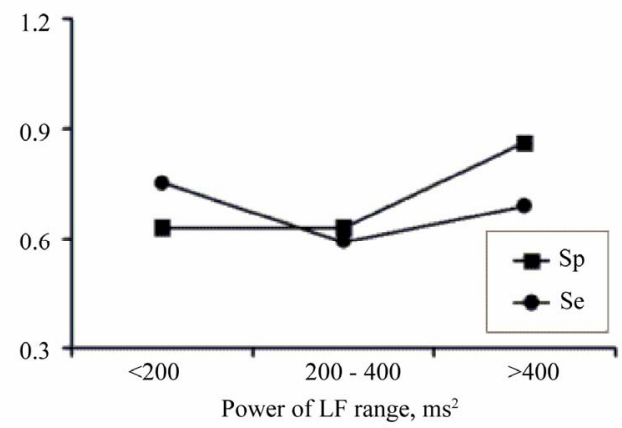

(a)

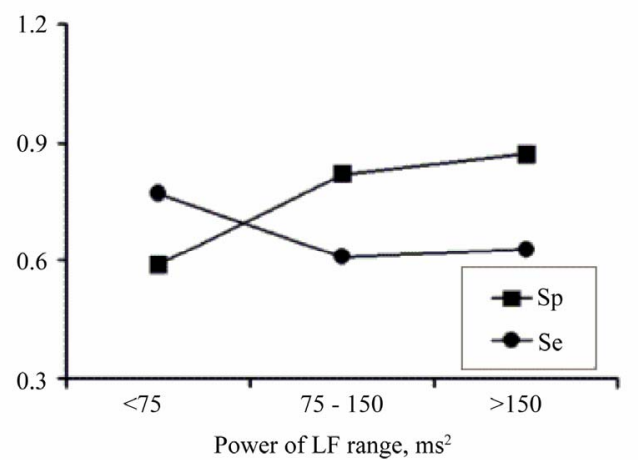

(b)

Figure 2. Assessments of $S e$ and $S p$ of bicycle ergometry in CHD patients based LF range power in rest (a) and load of $25 \mathrm{~W}$ (b). 

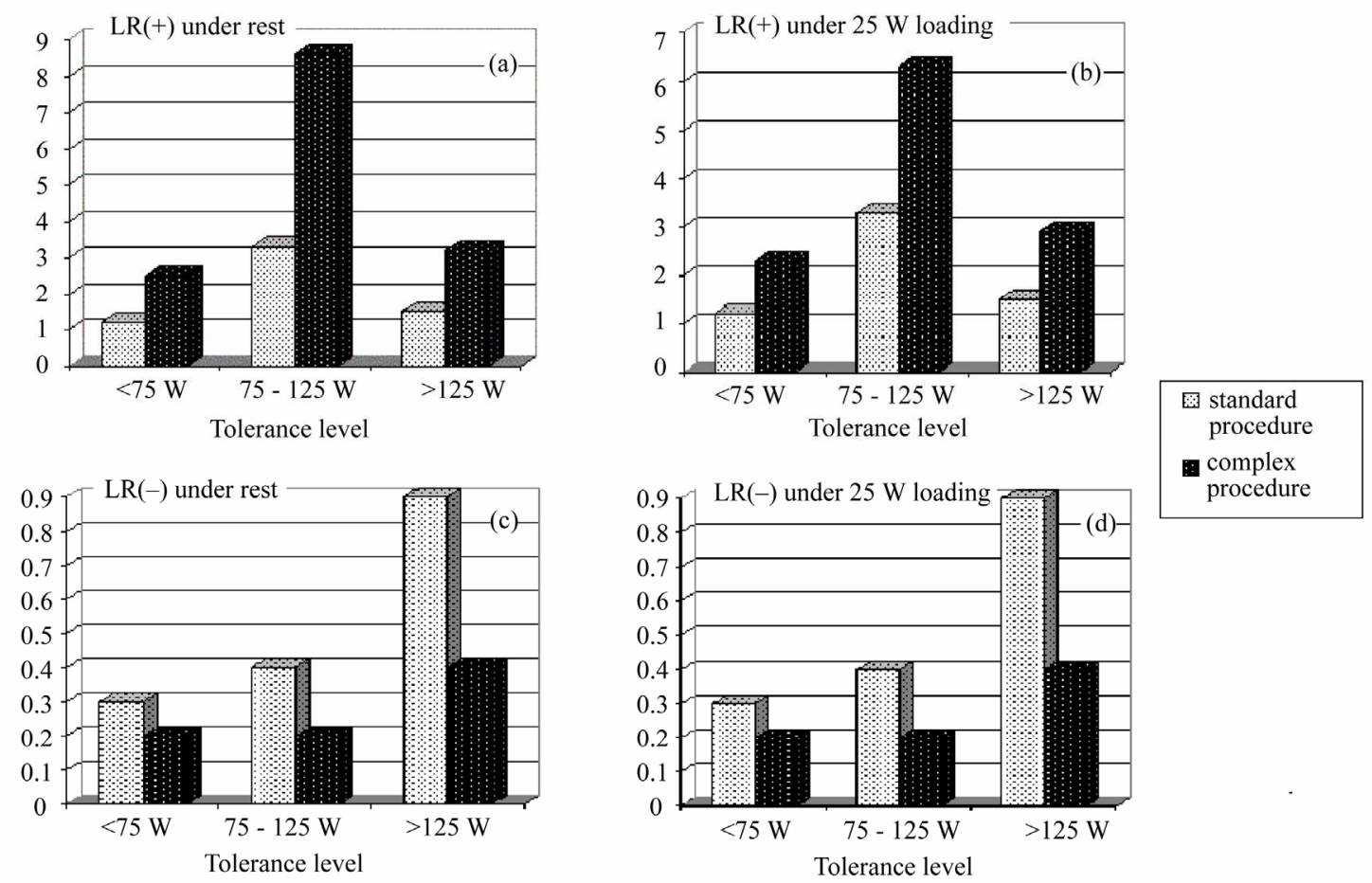

Figure 3. Likelihood ratio (LR) of standard procedure of bicycle ergometry results interpretation and likelihood ratio (LR) of complex assessment of bicycle ergometry results (tolerance level and the power of LF range of HRV spectrum) under the rest and $25 \mathrm{~W}$ loading. (a) LR(+) under rest; (b) LR(+) under $25 \mathrm{~W}$ loading; (c) LR(-) under rest; (d) LR(-) under $25 \mathrm{~W}$ loading.

Table 2. Likelihood ratio (LR) the bicycle ergometry results in CHD patients for the LF range spectral power of HRV under the rest.

\begin{tabular}{|c|c|c|c|}
\hline \multirow{2}{*}{$\begin{array}{l}\text { LF range spectral } \\
\text { power, } \mathrm{ms}^{2}\end{array}$} & \multicolumn{3}{|c|}{ Load tolerance $(\mathrm{W})$} \\
\hline & $<75$ & $75 \div 125$ & $>125$ \\
\hline \multicolumn{4}{|c|}{ Positive result of bicycle ergometry } \\
\hline$<200 \mathrm{~ms}^{2}$ & 3.1 & 8.6 & 3.9 \\
\hline $200 \div 400 \mathrm{~ms}^{2}$ & 2.5 & 6.9 & 3.2 \\
\hline$>400 \mathrm{~ms}^{2}$ & 5.3 & 14.5 & 6.6 \\
\hline \multicolumn{4}{|c|}{ Negative result of bicycle ergometry } \\
\hline$<200 \mathrm{~ms}^{2}$ & 0.2 & 0.2 & 0.4 \\
\hline $200 \div 400 \mathrm{~ms}^{2}$ & 0.2 & 0.2 & 0.5 \\
\hline$>400 \mathrm{~ms}^{2}$ & 0.2 & 0.2 & 0.4 \\
\hline
\end{tabular}

Table 3. Likelihood ratio (LR) of bicycle ergometry results in CHD patients for the LF range spectral power of HRV under $25 \mathrm{~W}$ loading.

\begin{tabular}{cccc}
\hline $\begin{array}{c}\text { LF-range spectral } \\
\text { power, } \mathrm{ms}^{2}\end{array}$ & \multicolumn{3}{c}{ Load tolerance $(\mathrm{W})$} \\
\cline { 2 - 4 } & \multicolumn{3}{c}{ Positive result of bicycle ergometry } \\
$<75 \mathrm{~ms}^{2}$ & 2.3 & 6.3 & 2.9 \\
$75 \div 150 \mathrm{~ms}^{2}$ & 4.1 & 11.2 & 5.1 \\
$>150 \mathrm{~ms}^{2}$ & 5.8 & 15.8 & 7.2 \\
\multicolumn{4}{r}{ Negative result of bicycle ergometry } \\
$<75 \mathrm{~ms}^{2}$ & 0.2 & 0.2 & 0.4 \\
$75 \div 150 \mathrm{~ms}^{2}$ & 0.2 & 0.2 & 0.5 \\
$>150 \mathrm{~ms}^{2}$ & 0.2 & 0.2 & 0.4 \\
\hline
\end{tabular}

Probability of false-negative bicycle ergometry outcomes keeps on the level of standard procedure of testing results evaluation. It follows from this that any negative test result is characterized by low reliability and thus could not be used for the assessment of the severity of coronary pathology. It agrees with the European Association of Cardiology data showing that probability of CHD in a 65 years patient with true angina is more, then $75 \%$ even if the bicycle ergometry showed a negative result [18]. At the same time, the reliability of positive bicycle ergometry results could be assessed taking into consideration the load tolerance and the spectral power in LF range of HRV. As a criterion of the diagnostic value of the test results the likelihood ratio (LR) index could be used. This index gives direct knowledge of the probability of CHD in the presence of a positive or negative physical load test outcome. In the clinic of Saratov Institute of Cardiology LR+ values no less then 5 were used as a threshold for the positive result of exercise testing and LR- values less then 0.2 were used for the negative one. In the case of exceeding the likelihood ratio threshold values it is possible to consider that an exercise test result reliably conform to the clinical picture of illness. Otherwise, if LR values do not exceed the threshold ones, then a stress test result could be considered unreliable. 
The approach offered allows to determine bicycle ergometry result reliability in cases of positive or negative results of the test individually in each patient and decrease possibility of diagnostic mistakes. Limitations of the method offered for CHD diagnostics are initial heart rate increase (heart rate more then 100 beats per minute) and impossibility to obtain suitable for the spectral analysis bicycle ergometry rhythmograms due to noise and artifacts presence.

\section{CONCLUSIONS}

Thus, the analysis of spectral power of LF range of HRV allows to advance diagnostic value of bicycle ergometry results in CHD patients. It is necessary to use a complex assessment of bicycle ergometry results taking into account the load tolerance level and a power of LF range of HRV spectrum. Combined use of the load tolerance and LF range spectral power levels (in rest and during $25 \mathrm{~W}$ loading) the validity of an individual exercise raises 2 - 3 times (in particular, bicycle ergometry) test result compared with standard procedure of the bicycle ergometry results analysis in CHD patients.

Table 2 can be used for preliminary assessment of the bicycle ergometry results especially in CHD patients with low levels of LF range spectral power $\left(<400 \mathrm{~ms}^{2}\right)$. Further the obtained value of likelihood ratio can be specified with the help of Table 3. Table data are constructed for CHD patients with hemodynamically significant atherosclerotic stenosis at least one coronary vessel (stenosis degree $>50 \%$ according to data of coronarography).

\section{REFERENCES}

[1] European Society of Cardiology (1993) Guidelines for cardiac exercise testing: ESC working group on exercise physiology, physiopathology and electrocardiography. European Heart Journal, 14, 969-988.

[2] Porta, C. and Bernardi, L. (2001) Interaction between respiration, autonomic function, and respiratory pattern inheart failure. New possibilities of rehabilitation intervention. Italian Heart Journal, 2, 624-627.

[3] Juhani, K.E., Marku, J.I., Heikki, V.H., et al. (1993) Responses of heart rate variability to coronari occlusion during coronari angioplasty. American Journal of Cardiology, 72, 1026-1030. doi:10.1016/0002-9149(93)90857-9

[4] De Boer, R.W., Karemuker, J.M. and Stracker, J. (1986) On the spectral analysis of blood pressure variability. American Journal of Physiology, 251, 685-687.
[5] De Boer, R.W., Karemuker, J.M. and Stracker, J. (1985) Relationships between short-term blood pressure fluctuations and heart variability in resting subjects. II: A simple model. Medical \& Biological Engineering \& Computing, 23, 359-364. doi:10.1007/BF02441590

[6] De Boer, R.W., Karemuker, J.M. and Stracker, J. (1985) Relationships between short-term blood pressure fluctuations and heart variability in resting subjects. I: A spectral analysis approach. Medical \& Biological Engineering \& Computing, 23, 352-358. doi:10.1007/BF02441589

[7] De Boer, R.W., Karemuker, J.M. and Stracker, J. (1987) Hemodynamic fluctuations and baroreflex sensitivity in humans: A beat-to-beat model. American Journal of Physiology, 253, 680-687.

[8] Madwed, J.B., Albrecht, P., Mark, R.G. and Cohen, R.J. (1989) Low-frequency oscillation in arterial pressure and heart-rate: A simple computer model. American Journal of Physiology, 256, 1573-1579.

[9] Pagani, M. and Malliani, A. (2000) Interpreting oscillations of muscle sympathetic nerve activity and heart rate variability. Journal of Hypertension, 18, 1709-1719. doi:10.1097/00004872-200018120-00002

[10] Sleight, P., La Rovere, M.T., Mortara, A., et al. (1995) Physiology and pathophysiology of heart rate variability in humans: is power spectral analysis largely an index of baroreflex gain? Clinical Science, 88, 103-109.

[11] Richter, D.W. and Spyer, K.M. (1990) Cardiorespiratory control. Oxford University Press, New York.

[12] Cevese, A., Grasso, R., Poltronieri, R. and Schena, F. (1995) Vascular resistance and arterial pressure low-frequency oscillations in the anesthetized dog. American Journal of Physiology, 268, 7-16.

[13] Whittam, A.M., Claytont, R.H., Lord, S.W., et al. (2000) Heart rate and blood pressure variability in normal subjects compared with data from beat-to-beat models developed from de Boer's model of the cardiovascular system. Physiological Measuremen, 21, 305-318. doi:10.1088/0967-3334/21/2/310

[14] Bernardi, L., Passino, C., Spadacini, G., et al. (1997) Arterial baro-receptor as determinants of $0.1 \mathrm{~Hz}$ and respiration-related changes in blood pressure and heart rate spectra. IOS Press, Amsterdam.

[15] Simon, C.M. (2002) Neural influeces on cardiovascular variability: Possibilities and pitfalls. The American Physiological Society, 282, 6-20.

[16] Andersen, K., Shephard, R., Denolin, H., et al. (1979) Fundamentals of exercise testing. WHO, Geneva.

[17] American Heart Association (1996) Heart rate variability. Standarts of measurement, physiological interpretation and clinical use. Circulation, 93, 1043-1065. doi:10.1161/01.CIR.93.5.1043

[18] European Society of Cardiology (1997) Management of stable angina pectoris: Recommendations of the task force of the European Society of Cardiology. European Heart Journal, 18, 394-413. 